\title{
Nontrivial solutions of a class of fractional differential equations with $p$-Laplacian via variational methods
}

\author{
Yan Qiao', Fangqi Chen ${ }^{1,2^{*}}$ and Yukun $\mathrm{An}^{1 *}$
}

\section{"Correspondence:}

fangqichen1963@126.com; anykna@nuaa.edu.cn

'Department of Mathematics,

Nanjing University of Aeronautics

and Astronautics, Nanjing, China

${ }^{2}$ College of Mathematics and

Systems Science, Shandong

University of Science and

Technology, Qingdao, China

\begin{abstract}
In this paper, a class of boundary value problems for fractional differential equations with a parameter is studied via the variational methods. Firstly, we present a result that the boundary value problems have at least one weak solution under the quadratic condition and the superquadratic condition, respectively. Additionally, we obtain the existence of at least one nontrivial solution by using the famous mountain pass lemma without the Ambrosetti-Rabinowitz condition. Finally, by a recent critical points theorem of Bonanno and Marano, the existence of at least three solutions is established.
\end{abstract}

MSC: $34 \mathrm{~A} 08 ; 35 \mathrm{D} 30$

Keywords: Fractional differential equations; Boundary value problem; Critical point theorem; Nontrivial solutions; Existence and multiplicity

\section{Introduction}

Fractional-order derivatives and integrals are more suitable to describe the properties of real materials than those of integer-order, so fractional-order differential equations are more and more widely used in simulating the mechanical and electrical characteristics of real materials, dynamic system control theory, rock rheological properties, and many other fields (see [1-10] and their references). The existence of solutions for boundary value problems of fractional differential equations is also studied in various ways, such as some fixed point theorems, the fixed point index theory in cones, methods of upper and lower solutions, coincidence degree theory, topological degree theory, etc. (see [11-16]). Later, with the introduction of the mountain pass theorem, variational methods have become a new and effective tool to study the existence of solutions for boundary value problems (see [17-19] and their references). In addition, nonlinear terms are often required to satisfy the Ambrosetti-Rabinowitz (A-R for short) condition when variational methods are applied. For example, in [20], Zhao and Tang studied a class of boundary value problems for fractional differential equations and obtained the existence of at least one weak solution by using the critical point theory under satisfying the A-R condition.

(c) The Author(s) 2020. This article is licensed under a Creative Commons Attribution 4.0 International License, which permits use sharing, adaptation, distribution and reproduction in any medium or format, as long as you give appropriate credit to the original author(s) and the source, provide a link to the Creative Commons licence, and indicate if changes were made. The images or other third party material in this article are included in the article's Creative Commons licence, unless indicated otherwise in a credit line to the material. If material is not included in the article's Creative Commons licence and your intended use is not permitted by statutory regulation or exceeds the permitted use, you will need to obtain permission directly from the copyright holder. To view a copy of this licence, visit http://creativecommons.org/licenses/by/4.0/. 
Chen and Liu [21] considered the following boundary value problems of fractional differential equations and obtained the existence of at least one weak solution under the A-R condition:

$$
\left\{\begin{array}{l}
{ }_{t} D_{T}^{\alpha} \phi_{p}\left({ }_{0} D_{t}^{\alpha} u(t)\right)=f(t, u(t)), \quad t \in[0, T], \\
u(0)=u(T)=0
\end{array}\right.
$$

where ${ }_{0} D_{t}^{\alpha}$ and ${ }_{t} D_{T}^{\alpha}$ are the left and right Riemann-Liouville fractional derivatives of order $\alpha \in(0,1]$ respectively. $f:[0, T] \times \mathbb{R} \rightarrow \mathbb{R}$. The result is as follows.

Theorem 1.1 ([21], Theorem 4.2) Let $\frac{1}{p}<\alpha \leq 1$. Assume that

$\left(V_{1}\right) f \in C([0, T] \times \mathbb{R}, \mathbb{R})$.

$\left(V_{2}\right)$ There exist constants $\mu \in\left(0, \frac{1}{p}\right)$ and $M>0$ such that

$$
0<F(t, x) \leq \mu x f(t, x), \quad \forall t \in[0, T], x \in \mathbb{R} \text { with }|x| \geq M
$$

$\left(V_{3}\right)$ For $t \in[0, T]$ and $x \in \mathbb{R}$, we have

$$
\limsup _{|x| \rightarrow 0} \frac{F(t, x)}{|x|^{p}}<\frac{(\Gamma(\alpha+1))^{p}}{p T^{\alpha p}} .
$$

Then BVP (1.1) admits at least one nontrivial weak solution.

Motivated by the above mentioned works, we are interested in the following fractional differential boundary value problem:

$$
\left\{\begin{array}{l}
{ }_{t} D_{T}^{\alpha} \phi_{p}\left({ }_{0} D_{t}^{\alpha} x(t)\right)=\lambda f(t, x(t)), \quad \text { a.e. } t \in[0, T], \\
x(0)=x(T)=0,
\end{array}\right.
$$

where $0<\alpha \leq 1,{ }_{0} D_{t}^{\alpha}$ and ${ }_{t} D_{T}^{\alpha}$ denote the left and right Riemann-Liouville fractional derivatives of order $\alpha$, respectively. $\lambda>0$ is a parameter. $\phi_{p}: \mathbb{R} \rightarrow \mathbb{R}$ is the $p$-Laplacian defined by $\phi_{p}(s)=|s|^{p-2} s$ if $s \neq 0, \phi_{p}(0)=0,1<p<\infty$. $f:[0, T] \times \mathbb{R} \rightarrow \mathbb{R}$ and $F(t, x)=$ $\int_{0}^{x} f(t, s) \mathrm{d} s$. The existence and multiplicity of solutions for boundary value problem (BVP for short) (1.2) will be derived by the critical point theory.

In the following proofs, we assume that $f(t, x)$ and $F(t, x)$ satisfy some of the following conditions:

$\left(A_{0}\right) F(t, x)$ is measurable in $t$ for every $x \in \mathbb{R}$ and continuously differentiable in $x$ for a.e. $t \in[0, T]$, and there exist $k_{1} \in C\left(\mathbb{R}^{+}, \mathbb{R}^{+}\right)$and $k_{2} \in L^{1}\left([0, T], \mathbb{R}^{+}\right)$such that

$$
|F(t, x)| \leq k_{1}(|x|) k_{2}(t),|f(t, x)| \leq k_{1}(|x|) k_{2}(t)
$$

for all $x \in \mathbb{R}$ and a.e. $t \in[0, T]$.

$\left(A_{1}\right)$ There exist a constant $0 \leq \theta<p$ and a function $\eta_{1}(t) \in C([0, T])$ with essinf $\eta_{1}>0$ such that

$$
\limsup _{|x| \rightarrow \infty} \frac{F(t, x)}{|x|^{\theta}}<\eta_{1}(t)
$$

uniformly for a.e. $t \in[0, T]$. 
$\left(A_{2}\right)$ There is a function $\eta_{2}(t) \in C([0, T])$ with ess inf $\eta_{2}>0$ such that

$$
\limsup _{|x| \rightarrow \infty} \frac{F(t, x)}{|x|^{p}}<\eta_{2}(t)
$$

uniformly for a.e. $t \in[0, T]$.

$\left(A_{3}\right) \lim \sup _{|x| \rightarrow 0} \frac{F(t, x)}{|x|^{p}}=0$ uniformly for a.e. $t \in[0, T]$.

$\left(A_{4}\right) F(t, x) \geq 0$ for all $t \in[0, T]$ and $x \in \mathbb{R}$.

$\left(A_{5}\right)$ There is a constant $\vartheta>p$ such that

$$
\limsup _{|x| \rightarrow \infty} \frac{\vartheta F(t, x)-f(t, x) x}{|x|^{p}} \leq 0
$$

uniformly for a.e. $t \in[0, T]$.

$\left(A_{6}\right)$ There exists a subset $\Omega$ of $[0, T]$ with meas $(\Omega)>0$ such that

$$
\liminf _{|x| \rightarrow \infty} \frac{F(t, x)}{|x|^{p}}>0
$$

uniformly for a.e. $t \in \Omega$.

Remark 1.1 By $\left(A_{1}\right)$, there exists a constant $L_{1}>0$ such that

$$
F(t, x) \leq \eta_{1}(t)|x|^{\theta}+L_{1}
$$

for a.e. $t \in[0, T]$ and $x \in \mathbb{R}$.

Similarly, $\left(A_{2}\right)$ implies that there is a constant $L_{2}>0$ such that

$$
F(t, x) \leq \eta_{2}(t)|x|^{p}+L_{2}
$$

for a.e. $t \in[0, T]$ and $x \in \mathbb{R}$.

The following arrangement of the article is as follows. In the second part, the basic definitions, some properties and lemmas of fractional calculus are given. Additionally, the related fractional derivative space and the variational structure of BVP (1.2) is established. In the third part, we give the existence and multiplicity theorems of nontrivial solutions of BVP (1.2) under some appropriate conditions.

\section{Preliminaries}

In order to obtain the existence of solutions to BVP (1.2), we need to recall some necessary definitions and related properties of fractional calculus, which will be used in the proofs later in this paper.

Definition 2.1 $([22,23])$ Let $u$ be a function defined on $[a, b]$. The left and right RiemannLiouville fractional integrals of order $\gamma$ for the function $u$ denoted by ${ }_{a} D_{t}^{-\gamma} u(t)$ and ${ }_{t} D_{b}^{-\gamma} u(t)$, respectively, are defined by

$$
{ }_{a} D_{t}^{-\gamma} u(t)=\frac{1}{\Gamma(\gamma)} \int_{a}^{t}(t-s)^{\gamma-1} u(s) \mathrm{d} s, \quad t \in[a, b], \gamma>0
$$


and

$$
{ }_{t} D_{b}^{-\gamma} u(t)=\frac{1}{\Gamma(\gamma)} \int_{t}^{b}(s-t)^{\gamma-1} u(s) \mathrm{d} s, \quad t \in[a, b], \gamma>0
$$

provided the right-hand sides are pointwise defined on $[a, b]$, where $\Gamma>0$ is the gamma function.

Definition 2.2 $([22,23])$ Let $u$ be a function defined on $[a, b]$. The left and right RiemannLiouville fractional derivatives of order $\gamma$ for the function $u$ denoted by ${ }_{a} D_{t}^{\gamma} u(t)$ and ${ }_{t} D_{b}^{\gamma} u(t)$, respectively, are defined by

$$
{ }_{a} D_{t}^{\gamma} u(t)=\left({ }_{a} D_{t}^{\gamma-n} u(t)\right)^{(n)}=\frac{1}{\Gamma(n-\gamma)} \frac{\mathrm{d}^{n}}{\mathrm{~d} t^{n}}\left(\int_{a}^{t}(t-s)^{n-\gamma-1} u(s) \mathrm{d} s\right)
$$

and

$$
{ }_{t} D_{b}^{\gamma} u(t)=(-1)^{n}\left({ }_{t} D_{b}^{\gamma-n} u(t)\right)^{(n)}=\frac{1}{\Gamma(n-\gamma)}(-1)^{n} \frac{\mathrm{d}^{n}}{\mathrm{~d} t^{n}}\left(\int_{t}^{b}(s-t)^{n-\gamma-1} u(s) \mathrm{d} s\right),
$$

where $t \in[a, b], n-1 \leq \gamma<n$, and $n \in \mathbb{N}$.

Remark $2.1([22,23])$ If $0 \leq \gamma<1$, then

$$
{ }_{a} D_{t}^{\gamma} u(t)=\left({ }_{a} D_{t}^{\gamma-1} u(t)\right)^{\prime}=\frac{1}{\Gamma(1-\gamma)} \frac{\mathrm{d}}{\mathrm{d} t}\left(\int_{a}^{t}(t-s)^{-\gamma} u(s) \mathrm{d} s\right), \quad t \in[a, b]
$$

and

$$
{ }_{t} D_{b}^{\gamma} u(t)=-\left({ }_{t} D_{b}^{\gamma-1} u(t)\right)^{\prime}=-\frac{1}{\Gamma(1-\gamma)} \frac{\mathrm{d}}{\mathrm{d} t}\left(\int_{t}^{b}(s-t)^{-\gamma} u(s) \mathrm{d} s\right), \quad t \in[a, b] .
$$

Remark 2.2 ([19]) For $n \in \mathbb{N}$, if $\gamma=n-1$, according to Definition 2.2, we find that

$$
\begin{aligned}
& { }_{a} D_{t}^{n-1} u(t)=u^{(n-1)}(t), \\
& { }_{t} D_{b}^{n-1} u(t)=(-1)^{n-1} u^{(n-1)}(t),
\end{aligned}
$$

where $u^{(n-1)}(t)$ is the usual derivative of order $n-1$.

Now, we present the rule for fractional integration by parts.

Property 2.1 ([23]) If $u \in L^{p}\left([a, b], \mathbb{R}^{N}\right), v \in L^{q}\left([a, b], \mathbb{R}^{N}\right)$ and $p \geq 1, q \geq 1, \frac{1}{p}+\frac{1}{q} \leq 1+\gamma$ or $p \neq 1, q \neq 1, \frac{1}{p}+\frac{1}{q}=1+\gamma$. Then

$$
\int_{a}^{b}\left[{ }_{a} D_{t}^{-\gamma} u(t)\right] v(t) \mathrm{d} t=\int_{a}^{b}\left[{ }_{t} D_{b}^{-\gamma} v(t)\right] u(t) \mathrm{d} t, \quad \gamma>0 .
$$

In order to deal with BVP (1.2), we introduce appropriate function spaces and the related variational structure. 
Let $L^{p}([0, T], \mathbb{R})(1 \leq p<\infty)$ and $C([0, T], \mathbb{R})$ be the $p$-Lebesgue space and the continuous function space, respectively, with the norms

$$
\|u\|_{L^{p}}=\left(\int_{0}^{T}|u(t)|^{p} \mathrm{~d} t\right)^{\frac{1}{p}}, \quad u \in L^{p}([0, T], \mathbb{R})(1 \leq p<\infty)
$$

and

$$
\|u\|_{\infty}=\max _{t \in[0, T]}|u(t)|, \quad u \in C([0, T], \mathbb{R}) .
$$

To set up the variational structure of BVP (1.2), we need to construct the appropriate function space first.

Definition 2.3 Let $0<\alpha \leq 1$ and $1<p<\infty$. The fractional derivative space $E_{0}^{\alpha, p}$ is defined as the closure of $C_{0}^{\infty}([0, T], \mathbb{R})$, that is, $E_{0}^{\alpha, p}=\overline{C_{0}^{\infty}([0, T], \mathbb{R})}$ with the norm

$$
\|x\|_{\alpha, p}=\left(\int_{0}^{T}\left|{ }_{0} D_{t}^{\alpha} x(t)\right|^{p} \mathrm{~d} t+\int_{0}^{T}|x(t)|^{p} \mathrm{~d} t\right)^{\frac{1}{p}} .
$$

Remark 2.3 The fractional derivative space $E_{0}^{\alpha, p}$ is the space of functions $x(t) \in L^{p}([0, T], \mathbb{R})$ with an $\alpha$-order Riemann-Liouville fractional derivative ${ }_{0} D_{t}^{\alpha} x(t) \in L^{p}([0, T], \mathbb{R})$ and $x(0)=$ $x(T)=0$.

Remark 2.4 From [19], the fractional derivative space $E_{0}^{\alpha, p}(0<\alpha \leq 1,1<p<\infty)$ is a reflexive and separable Banach space.

Lemma 2.1 ([19]) Let $0<\alpha \leq 1$ and $1<p<\infty$. For any $x \in E_{0}^{\alpha, p}$, we have

$$
\|x\|_{L^{p}} \leq \frac{T^{\alpha}}{\Gamma(\alpha+1)}\left\|_{0} D_{t}^{\alpha} x\right\|_{L^{p}}
$$

If $\alpha>\frac{1}{p}$ and $\frac{1}{p}+\frac{1}{q}=1$, then

$$
\|x\|_{\infty} \leq \frac{T^{\alpha-\frac{1}{p}}}{\Gamma(\alpha)((\alpha-1) q+1)^{\frac{1}{q}}}\left\|_{0} D_{t}^{\alpha} x\right\|_{L^{p}} .
$$

We denote $G=\frac{T^{\alpha-\frac{1}{p}}}{\Gamma(\alpha)((\alpha-1) q+1)^{\frac{1}{q}}}$.

Remark 2.5 According to formula (2.2), let $x \in E_{0}^{\alpha, p}$, the norm $\|x\|_{\alpha, p}$ is equivalent to

$$
\|x\|_{\alpha, p}=\left(\int_{0}^{T}\left|{ }_{0} D_{t}^{\alpha} x(t)\right|^{p} \mathrm{~d} t\right)^{\frac{1}{p}}
$$

In the following discussion, we denote $\|x\|=\|x\|_{\alpha, p}$.

Lemma 2.2 ([19]) Let $0<\alpha \leq 1$ and $1<p<\infty$. Assume that $\alpha>\frac{1}{p}$ and the sequence $\left\{x_{n}\right\}$ converges weakly to $x$ in $E_{0}^{\alpha, p}$, that is, $x_{n} \rightarrow x$ as $n \rightarrow \infty$. Then $x_{n} \rightarrow x$ in $C([0, T], \mathbb{R})$ as $n \rightarrow \infty$, that is, $\left\|x_{n}-x\right\|_{\infty} \rightarrow 0$ as $n \rightarrow \infty$. 
Definition 2.4 The weak solution of BVP (1.2) is a function $x \in E_{0}^{\alpha, p}$ satisfying the following equation:

$$
\int_{0}^{T} \phi_{p}\left({ }_{0} D_{t}^{\alpha} x(t)\right)_{0} D_{t}^{\alpha} y(t) \mathrm{d} t=\lambda \int_{0}^{T} f(t, x(t)) y(t) \mathrm{d} t, \quad \forall y \in E_{0}^{\alpha, p} .
$$

For the sake of convenience, the solution of BVP (1.2) mentioned later is the weak solution of BVP (1.2).

For $\forall x \in E_{0}^{\alpha, p}$, we consider the functional $I: E_{0}^{\alpha, p} \rightarrow \mathbb{R}$ as follows:

$$
\begin{aligned}
I(x) & =\frac{1}{p} \int_{0}^{T}\left|{ }_{0} D_{t}^{\alpha} x(t)\right|^{p} \mathrm{~d} t-\lambda \int_{0}^{T} F(t, x(t)) \mathrm{d} t \\
& =\frac{1}{p}\|x\|^{p}-\lambda \int_{0}^{T} F(t, x(t)) \mathrm{d} t .
\end{aligned}
$$

From [25], we can know that $I \in C^{1}\left(E_{0}^{\alpha, p}, \mathbb{R}\right)$ and

$$
I^{\prime}(x) y=\int_{0}^{T} \phi_{p}\left({ }_{0} D_{t}^{\alpha} x(t)\right)_{0} D_{t}^{\alpha} y(t) \mathrm{d} t-\lambda \int_{0}^{T} f(t, x(t)) y(t) \mathrm{d} t, \quad \forall y \in E_{0}^{\alpha, p} .
$$

It follows from Definition 2.4 and (2.6) that if $x \in E_{0}^{\alpha, p}$ is a solution of $I^{\prime}(x)=0, x$ is a weak solution of BVP (1.2).

\section{Existence and multiplicity of solutions of BVP (1.2)}

Definition 3.1 Let $E$ be a real Banach space and $J \in C^{1}(E, \mathbb{R})$. If any sequence $\left\{x_{n}\right\} \subset E$ for which $\left\{\left(x_{n}\right)\right\}$ is bounded and $J^{\prime}\left(x_{n}\right) \rightarrow 0$ as $n \rightarrow+\infty$ possesses a convergent subsequence, then we say that $J$ satisfies the Palais-Smale condition (P.S. condition for short).

Lemma 3.1 ([24]) Let $E$ be a real reflexive Banach space. If the functional $J: E \rightarrow \mathbb{R}$ is weakly lower semicontinuous and coercive, i.e., $\lim _{\|x\| \rightarrow \infty} J(x)=+\infty$, then there exists $x_{*} \in E$ such that $J\left(x_{*}\right)=\inf _{x \in E} J(x)$. Moreover, if $J$ is also Fréchet differentiable on E, then $J^{\prime}\left(x_{*}\right)=0$.

Lemma 3.2 ([25] Mountain pass theorem) Let $E$ be a real Banach space and $J \in C^{1}(E, \mathbb{R})$ satisfy the P.S. condition. Suppose that

(1) $J(0)=0$.

(2) There exist $\rho>0$ and $\sigma>0$ such that $J\left(x_{0}\right) \geq \sigma$ for all $x_{0} \in E$ with $\left\|x_{0}\right\|=\rho$.

(3) There exists $x_{1} \in E$ with $\left\|x_{1}\right\| \geq \rho$ such that $J\left(x_{1}\right)<\sigma$.

Then $z=\inf _{h \in \Delta} \max _{t \in[0,1]} J(h(t)) \geq \sigma$ is a critical value of $J$, where

$$
\Delta=\left\{h \in C([0,1], E) \mid h(0)=x_{0}, h(1)=x_{1}\right\} .
$$

Lemma 3.3 ([26]) Let E be a real reflexive Banach space, $\Phi: E \rightarrow \mathbb{R}$ be a sequentially weakly lower semicontinuous, coercive, and continuously Gâteaux differentiable functional whose Gâteaux derivative admits a continuous inverse on $E^{*}, \Psi: E \rightarrow \mathbb{R}$ be a continuously Gâteaux differentiable functional whose Gâteaux derivative is compact such that

$$
\inf _{x \in E} \Phi(x)=\Phi(0)=\Psi(0)=0
$$


(1) $\sup \{\Psi(x): \Phi(x) \leq r\}<r \frac{\Psi(\omega)}{\Phi(\omega)}$

(2) for all $\lambda \in\left(\frac{\Phi(\omega)}{\Psi(\omega)}, \frac{r}{\sup \{\Psi(x): \Phi(x) \leq r\}}\right)$, the functional $\Phi-\lambda \Psi$ is coercive.

Then, for each $\lambda \in\left(\frac{\Phi(\omega)}{\Psi(\omega)}, \frac{r}{\sup \{\Psi(x): \Phi(x) \leq r\}}\right)$, the functional $\Phi-\lambda \Psi$ has at least three distinct critical points in $E$.

Lemma 3.4 Suppose that $\left(A_{0}\right)$ and $\left(A_{4}-A_{5}\right)$ hold, then I satisfies the P.S. condition, i.e., for every sequence $\left\{x_{n}\right\} \in E_{0}^{\alpha, p},\left\{x_{n}\right\}$ has a convergent subsequence if

$$
\left\{I\left(x_{n}\right)\right\} \text { is bounded and } I^{\prime}\left(x_{n}\right) \rightarrow 0 \text { as } n \rightarrow \infty .
$$

Proof First we prove that $\left\{x_{n}\right\}$ is a bounded sequence in $E_{0}^{\alpha, p}$.

Without loss of generality, in the next proof of the theorem, the sequence $\left\{x_{n}\right\}$ and its subsequences are all denoted as $\left\{x_{n}\right\}$.

Suppose that $\left\{x_{n}\right\}$ is unbounded. Passing to a subsequence, we may assume that $\left\|x_{n}\right\| \rightarrow$ $\infty$. Let $\varpi_{n}=\frac{x_{n}}{\left\|x_{n}\right\|}$, so that $\left\|\varpi_{n}\right\|=1$. By Lemma 2.2, also passing to a subsequence, we can suppose that

$$
\begin{array}{ll}
\varpi_{n} \rightarrow \varpi_{0} & \text { weakly in } E_{0}^{\alpha, p}, \\
\varpi_{n} \rightarrow \varpi_{0} & \text { strongly in } C([0, T], \mathbb{R})
\end{array}
$$

as $n \rightarrow \infty$. Moreover, we have

$$
\bar{\varpi}_{n}=\frac{1}{T} \int_{0}^{T} \varpi_{n}(t) \mathrm{d} t \rightarrow \frac{1}{T} \int_{0}^{T} \varpi_{0}(t) \mathrm{d} t=\bar{\varpi}_{0}
$$

as $n \rightarrow \infty$. By (3.1) there exists a constant $K_{1}>0$ such that

$$
\begin{aligned}
\left(\frac{\vartheta}{p}-1\right)\left\|x_{n}\right\|^{p} & =\vartheta I\left(x_{n}\right)-I^{\prime}\left(x_{n}\right) x_{n}+\lambda \int_{0}^{T}\left(\vartheta F\left(t, x_{n}(t)\right)-f\left(t, x_{n}(t)\right) x_{n}(t)\right) \mathrm{d} t \\
& \leq K_{1}\left(1+\left\|x_{n}\right\|\right)+\lambda \int_{0}^{T}\left(\vartheta F\left(t, x_{n}(t)\right)-f\left(t, x_{n}(t)\right) x_{n}(t)\right) \mathrm{d} t .
\end{aligned}
$$

Notice that $\left\|x_{n}\right\| \rightarrow \infty$, we have

$$
\left(\frac{\vartheta}{p}-1\right)\left\|\varpi_{n}\right\|^{p} \leq \frac{K_{1}\left(1+\left\|x_{n}\right\|\right)}{\left\|x_{n}\right\|^{p}}+\lambda \frac{\int_{0}^{T}\left(\vartheta F\left(t, x_{n}(t)\right)-f\left(t, x_{n}(t)\right) x_{n}(t)\right) \mathrm{d} t}{\left\|x_{n}\right\|^{p}} .
$$

In view of $\left(A_{0}\right)$ and $\left(A_{5}\right)$, there exists $\Omega_{0} \subset[0, T]$ with meas $\left(\Omega_{0}\right)=0$ such that

$$
|F(t, x)| \leq k_{1}(|x|) k_{2}(t), \quad|f(t, x)| \leq k_{1}(|x|) k_{2}(t)
$$

for all $x \in \mathbb{R}$ and $t \in[0, T] \backslash \Omega_{0}$ and

$$
\limsup _{|x| \rightarrow \infty} \frac{\vartheta F(t, x)-f(t, x) x}{|x|^{p}} \leq 0
$$

uniformly for $t \in[0, T] \backslash \Omega_{0}$. This yields

$$
\limsup _{n \rightarrow \infty} \frac{\vartheta F\left(t, x_{n}(t)\right)-f\left(t, x_{n}(t)\right) x_{n}(t)}{\left\|x_{n}\right\|^{p}} \leq 0
$$


for $t \in[0, T] \backslash \Omega_{0}$. Otherwise, there exist $t_{0} \in[0, T] \backslash \Omega_{0}$ and a subsequence of $\left\{x_{n}\right\}$ such that

$$
\limsup _{n \rightarrow \infty} \frac{\vartheta F\left(t_{0}, x_{n}\left(t_{0}\right)\right)-f\left(t_{0}, x_{n}\left(t_{0}\right)\right) x_{n}\left(t_{0}\right)}{\left\|x_{n}\right\|^{p}}>0 .
$$

If $\left\{x_{n}\left(t_{0}\right)\right\}$ is bounded, then there exists a positive constant $K_{2}$ such that $\left|x_{n}\left(t_{0}\right)\right| \leq K_{2}$ for all $n \in \mathbb{N}$. By (3.4) we find

$$
\frac{\vartheta F\left(t_{0}, x_{n}\left(t_{0}\right)\right)-f\left(t_{0}, x_{n}\left(t_{0}\right)\right) x_{n}\left(t_{0}\right)}{\left\|x_{n}\right\|^{p}} \leq \frac{\left(\vartheta+K_{2}\right) \max _{s \in\left[0, K_{2}\right]} k_{1}(s) k_{2}\left(t_{0}\right)}{\left\|x_{n}\right\|^{p}} \rightarrow 0
$$

as $n \rightarrow \infty$, which contradicts (3.6). So, there is a subsequence of $\left\{x_{n}\left(t_{0}\right)\right\}$ such that $\left|x_{n}\left(t_{0}\right)\right| \rightarrow \infty$ as $n \rightarrow \infty$.

$$
\begin{aligned}
& \limsup _{n \rightarrow \infty} \frac{\vartheta F\left(t_{0}, x_{n}\left(t_{0}\right)\right)-f\left(t_{0}, x_{n}\left(t_{0}\right)\right) x_{n}\left(t_{0}\right)}{\left\|x_{n}\right\|^{p}} \\
& \quad=\limsup _{n \rightarrow \infty} \frac{\vartheta F\left(t_{0}, x_{n}\left(t_{0}\right)\right)-f\left(t_{0}, x_{n}\left(t_{0}\right)\right) x_{n}\left(t_{0}\right)}{\left|x_{n}\left(t_{0}\right)\right|^{p}}\left|\varpi_{n}\left(t_{0}\right)\right|^{p} \\
& \quad=\limsup _{n \rightarrow \infty} \frac{\vartheta F\left(t_{0}, x_{n}\left(t_{0}\right)\right)-f\left(t_{0}, x_{n}\left(t_{0}\right)\right) x_{n}\left(t_{0}\right)}{\left|x_{n}\left(t_{0}\right)\right|^{p}} \lim _{n \rightarrow \infty}\left|\varpi_{n}\left(t_{0}\right)\right|^{p} \leq 0 .
\end{aligned}
$$

This contradicts (3.6). Thus, (3.5) holds. From (3.3) and (3.5) we obtain

$$
\limsup _{n \rightarrow \infty}\left(\frac{\vartheta}{p}-1\right)\left\|\varpi_{n}\right\|^{p} \leq 0
$$

Since $\vartheta>p$, we get

$$
\left\|\varpi_{n}\right\|^{p} \rightarrow 0 \quad \text { as } n \rightarrow \infty
$$

but $\left\|\varpi_{n}\right\|=1$. This is a contradiction. Hence, $\left\{x_{n}\right\}$ is a bounded sequence in $E_{0}^{\alpha, p}$.

Since $E_{0}^{\alpha, p}$ is a reflexive space, there exists a weakly convergent subsequence such that $x_{n} \rightarrow x$ in $E_{0}^{\alpha, p}$. Hence, we have

$$
\begin{aligned}
& \left\langle I^{\prime}\left(x_{n}\right)-I^{\prime}(x), x_{n}-x\right\rangle \\
& \quad=\left\langle I^{\prime}\left(x_{n}\right), x_{n}-x\right\rangle-\left\langle I^{\prime}(x), x_{n}-x\right\rangle \\
& \quad \leq\left\|I^{\prime}\left(x_{n}\right)\right\|_{*}\left\|x_{n}-x\right\|-\left\langle I^{\prime}(x), x_{n}-x\right\rangle \\
& \quad \rightarrow 0
\end{aligned}
$$

as $n \rightarrow \infty$. Combining Lemma 2.2 with (2.3), we obtain that $\left\{x_{n}\right\}$ converges to $x$ strongly in $C([0, T], \mathbb{R})$, i.e., $\left\|x_{n}-x\right\| \rightarrow 0$ as $n \rightarrow \infty$, which implies that

$$
\int_{0}^{T}\left(f\left(t, x_{n}(t)\right)-f(t, x(t))\right)\left(x_{n}(t)-x(t)\right) \mathrm{d} t \rightarrow 0
$$

as $n \rightarrow \infty$. Note that

$$
\left\langle I^{\prime}\left(x_{n}\right)-I^{\prime}(x), x_{n}-x\right\rangle
$$




$$
\begin{aligned}
= & \int_{0}^{T}\left(\phi_{p}\left({ }_{0} D_{t}^{\alpha} x_{n}(t)\right)-\phi_{p}\left({ }_{0} D_{t}^{\alpha} x(t)\right)\right)\left({ }_{0} D_{t}^{\alpha} x_{n}(t)-{ }_{0} D_{t}^{\alpha} x(t)\right) \mathrm{d} t \\
& -\lambda \int_{0}^{T}\left(f\left(t, x_{n}(t)\right)-f(t, x(t))\right)\left(x_{n}(t)-x(t)\right) \mathrm{d} t .
\end{aligned}
$$

Thus, from (3.8) and (3.9) we have

$$
\int_{0}^{T}\left(\phi_{p}\left({ }_{0} D_{t}^{\alpha} x_{n}(t)\right)-\phi_{p}\left({ }_{0} D_{t}^{\alpha} x(t)\right)\right)\left({ }_{0} D_{t}^{\alpha} x_{n}(t)-{ }_{0} D_{t}^{\alpha} x(t)\right) \mathrm{d} t \rightarrow 0
$$

as $n \rightarrow \infty$. For any $s_{1}, s_{2} \in \mathbb{R}$, it is well known that (see Lemma 4.2 in [27]) there exists $a_{1}>0$ such that

$$
\left\langle\left|s_{1}\right|^{p-2} s_{1}-\left|s_{2}\right|^{p-2} s_{2}, s_{1}-s_{2}\right| \geq \begin{cases}a_{1}\left|s_{1}-s_{2}\right|^{p}, & p \geq 2, \\ a_{1} \frac{\left|s_{1}-s_{2}\right|^{2}}{\left(\left|s_{1}\right|+\left|s_{2}\right|\right)^{2-p}}, & 1<p \leq 2 .\end{cases}
$$

Following (3.11), we obtain that there exist $c_{1}, c_{2}>0$ such that

$$
\begin{aligned}
& \int_{0}^{T}\left(\phi_{p}\left({ }_{0} D_{t}^{\alpha} x_{n}(t)\right)-\phi_{p}\left({ }_{0} D_{t}^{\alpha} x(t)\right)\right)\left({ }_{0} D_{t}^{\alpha} x_{n}(t)-{ }_{0} D_{t}^{\alpha} x(t)\right) \mathrm{d} t \\
& \quad \geq \begin{cases}\left.c_{1} \int_{0}^{T}\right|_{0} D_{t}^{\alpha} x_{n}(t)-\left.{ }_{0} D_{t}^{\alpha} x(t)\right|^{p} \mathrm{~d} t, & p \geq 2, \\
c_{2} \int_{0}^{T} \frac{{ }_{0} D_{t}^{\alpha} x_{n}(t)-\left.D_{0} D_{t}^{\alpha} x(t)\right|^{2}}{\left({ }_{0} D_{t}^{\alpha} x_{n}(t) \mid+{ }_{0} D_{t}^{\alpha} x(t)\right)^{2-p}} \mathrm{~d} t, & 1<p<2 .\end{cases}
\end{aligned}
$$

When $1<p<2$, one has

$$
\begin{aligned}
& \int_{0}^{T}\left|{ }_{0} D_{t}^{\alpha} x_{n}(t)-{ }_{0} D_{t}^{\alpha} x(t)\right|^{p} \mathrm{~d} t \\
& \quad \leq\left(\int_{0}^{T} \frac{{ }_{0} D_{t}^{\alpha} x_{n}(t)-\left.{ }_{0} D_{t}^{\alpha} x(t)\right|^{2}}{\left({ }_{0} D_{t}^{\alpha} x_{n}(t)\left|+{ }_{0} D_{t}^{\alpha} x(t)\right|\right)^{2-p}} \mathrm{~d} t\right)^{\frac{p}{2}}\left(\int_{0}^{T}\left(\left|{ }_{0} D_{t}^{\alpha} x_{n}(t)\right|+\left|{ }_{0} D_{t}^{\alpha} x(t)\right|\right)^{p} \mathrm{~d} t\right)^{\frac{2-p}{2}} .
\end{aligned}
$$

Thus, noting that $\left(b_{1}+b_{2}\right)^{\gamma} \leq 2^{\gamma-1}\left(b_{1}^{\gamma}+b_{2}^{\gamma}\right)$, where $b_{1}, b_{2} \geq 0, \gamma \geq 1$ (see [28]), we have

$$
\begin{aligned}
& \int_{0}^{T}\left|{ }_{0} D_{t}^{\alpha} x_{n}(t)-{ }_{0} D_{t}^{\alpha} x(t)\right|^{p} \mathrm{~d} t \\
& \quad \leq c_{3}\left(\left\|x_{n}\right\|^{p}+\|x\|^{p}\right)^{\frac{2-p}{2}}\left(\int_{0}^{T} \frac{\left|{ }_{0} D_{t}^{\alpha} x_{n}(t)-{ }_{0} D_{t}^{\alpha} x(t)\right|^{2}}{\left(\left.\right|_{0} D_{t}^{\alpha} x_{n}(t)\left|+{ }_{0} D_{t}^{\alpha} x(t)\right|\right)^{2-p}} \mathrm{~d} t\right)^{\frac{p}{2}},
\end{aligned}
$$

where $c_{3}=2^{\frac{(p-1)(2-p)}{2}}$, which, together with (3.12), implies

$$
\begin{gathered}
\int_{0}^{T}\left(\phi_{p}\left({ }_{0} D_{t}^{\alpha} x_{n}(t)\right)-\phi_{p}\left({ }_{0} D_{t}^{\alpha} x(t)\right)\right)\left({ }_{0} D_{t}^{\alpha} x_{n}(t)-{ }_{0} D_{t}^{\alpha} x(t)\right) \mathrm{d} t \\
\quad \geq c_{2} c_{3}{ }_{3}^{-\frac{2}{p}}\left(\left\|x_{n}\right\|^{p}+\|x\|^{p}\right)^{\frac{p-2}{p}}\left\|x_{n}-x\right\|^{2}, \quad 1<p<2 .
\end{gathered}
$$

When $p \geq 2$, by (3.12) we get

$$
\int_{0}^{T}\left(\phi_{p}\left({ }_{0} D_{t}^{\alpha} x_{n}(t)\right)-\phi_{p}\left({ }_{0} D_{t}^{\alpha} x(t)\right)\right)\left({ }_{0} D_{t}^{\alpha} x_{n}(t)-{ }_{0} D_{t}^{\alpha} x(t)\right) \mathrm{d} t
$$




$$
\geq c_{1}\left\|x_{n}-x\right\|^{p}, \quad p \geq 2
$$

It follows from (3.10), (3.13), and (3.14) that

$$
\left\|x_{n}-x\right\| \rightarrow 0
$$

as $n \rightarrow \infty$, that is, $\left\{x_{n}\right\}$ converges strongly to $x$ in $E_{0}^{\alpha, p}$. The proof is completed.

Theorem 3.1 Assume that $\left(A_{0}\right)$ and one of the following conditions hold, then BVP (1.2) admits at least one nontrivial solution.

(1) $\left(A_{1}\right)$ holds and $\lambda \in(0, \infty)$.

(2) $\left(A_{2}\right)$ holds and $\lambda \in\left(0, \frac{1}{p G^{p} \int_{0}^{T} \eta_{2}(t) \mathrm{d} t}\right)$.

Proof We shall prove this theorem by Lemma 3.1.

Notice that

$$
\liminf _{n \rightarrow \infty}\left\|x_{n}\right\| \geq\|x\|
$$

Let $\left\{x_{n}\right\}$ converge weakly to $x$ in $E_{0}^{\alpha, p}$, then $x_{n}$ converges uniformly to $x$ in $[0, T]$. Therefore

$$
\begin{aligned}
\liminf _{n \rightarrow \infty} I\left(x_{n}\right) & =\liminf _{n \rightarrow \infty}\left\{\frac{1}{p}\left\|x_{n}\right\|^{p}-\lambda \int_{0}^{T} F\left(t, x_{n}(t)\right) \mathrm{d} t\right\} \\
& \geq \frac{1}{p}\|x\|^{p}-\lambda \int_{0}^{T} F(t, x(t)) \mathrm{d} t \\
& =I(x),
\end{aligned}
$$

which means that $I(x)$ is weakly lower semicontinuous.

If $\left(A_{1}\right)$ holds, it follows from (1.3) and (2.5) that

$$
\begin{aligned}
I(x) & =\frac{1}{p}\|x\|^{p}-\lambda \int_{0}^{T} F(t, x(t)) \mathrm{d} t \\
& \geq \frac{1}{p}\|x\|^{p}-\lambda G^{\theta}\|x\|^{\theta} \int_{0}^{T} \eta_{1}(t) \mathrm{d} t-\lambda L_{1} T .
\end{aligned}
$$

Because of $0 \leq \theta<p$, we obtain that $I(x) \rightarrow \infty$ as $\|x\| \rightarrow \infty$, which indicates that $I$ is coercive. Therefore, by Lemma 3.1, there is $x_{0} \in E_{0}^{\alpha, p}$ such that $I\left(x_{0}\right)=\inf _{x \in E_{0}^{\alpha, p}} I(x)$ and $I^{\prime}\left(x_{0}\right)=0$.

In the same way, if $\left(A_{2}\right)$ holds, according to (1.4) and (2.5), we have

$$
I(x) \geq \frac{1}{p}\|x\|^{p}-\lambda G^{p}\|x\|^{p} \int_{0}^{T} \eta_{2}(t) \mathrm{d} t-\lambda L_{2} T .
$$

On account of $0<\lambda<\frac{1}{p G^{p} \int_{0}^{T} \eta_{2}(t) \mathrm{d} t}$, then $I(x) \rightarrow \infty$ as $\|x\| \rightarrow \infty$ is the natural result. The proof is finished. 


\section{Remark 3.1}

(1) By $\left(A_{3}\right)$, we know that, for any $0<\epsilon<\frac{1}{\lambda p T G^{p}}$, there exists $\delta \in(0, \epsilon)$ such that

$$
|F(t, x)| \leq \epsilon|x|^{p}
$$

for a.e. $t \in[0, T]$ and $x \in \mathbb{R}$ with $|x| \leq \delta$.

(2) By $\left(A_{5}\right)$ and $\left(A_{6}\right)$ there exist constants $K_{3}>1, \varrho>\frac{(\vartheta-p) G^{p}}{2 \lambda p}$ and a subset of $\Omega$, still denoted by $\Omega$, with $|\Omega|=$ meas $(\Omega)>0$ such that

$$
\vartheta F(t, x)-f(t, x) x \leq \varrho|x|^{p}
$$

and

$$
F(t, x)>\frac{2 \varrho}{\vartheta-p}|x|^{p}
$$

for all $|x| \geq K_{3}$ and $t \in \Omega$.

It follows from $\left(A_{0}\right)$ that

$$
F(t, x) \leq \max _{s \in\left[0, K_{3}\right]} k_{1}(s) k_{2}(t)
$$

for all $|x| \leq K_{3}$ and a.e. $t \in[0, T]$.

Therefore, we obtain

$$
F(t, x)>\frac{2 \varrho}{\vartheta-p}\left(|x|^{p}-K_{3}^{p}\right)-\max _{s \in\left[0, K_{3}\right]} k_{1}(s) k_{2}(t)
$$

for all $|x| \in \mathbb{R}$ and $t \in \Omega$.

Theorem 3.2 If $\left(A_{0}\right)$ and $\left(A_{3}-A_{6}\right)$ hold, then BVP (1.2) admits at least one nontrivial weak solution for each $\lambda \in\left(0, \frac{1}{p \in T G^{p}}\right)$.

Proof Obviously, it follows from the definition of $I$ that $I(0)=0$. Therefore, (1) of Lemma 3.2 is true.

Let

$$
0<\rho=\frac{\delta}{G} \quad(\delta \text { is defined by Remark 3.1), }
$$

then it follows from (2.3) that

$$
\|x\|_{\infty} \leq G\|x\|=G \rho=\delta
$$

for all $x \in E_{0}^{\alpha, p}$ with $\|x\|=\rho$.

Therefore, for all $x \in E_{0}^{\alpha, p}$ with $\|x\|=\rho=\frac{\delta}{G}$, from Remark 3.1, we have

$$
I(x)=\frac{1}{p}\|x\|^{p}-\lambda \int_{0}^{T} F(t, x(t)) \mathrm{d} t
$$




$$
\begin{aligned}
& \geq \frac{1}{p}\|x\|^{p}-\lambda \epsilon T G^{p}\|x\|^{p} \\
& \geq\left(\frac{1}{p}-\lambda \epsilon T G^{p}\right) \frac{\delta^{p}}{G^{p}} \\
& =\sigma .
\end{aligned}
$$

In view of $0<\lambda<\frac{1}{p \in T G^{p}}$, we obtain that $\sigma>0$. This implies that (2) in Lemma 3.2 is satisfied.

Choose $x_{0}(t) \in E_{0}^{\alpha, p}$ with $\left\|x_{0}\right\| \leq G$ and $\int_{\Omega}\left|x_{0}(t)\right|^{p} \mathrm{~d} t=1$.

And for $\varsigma>0$, we have

$$
\begin{aligned}
I\left(\varsigma u_{0}\right) & =\frac{1}{p}\left\|\varsigma x_{0}\right\|^{p}-\lambda \int_{0}^{T} F\left(t, \varsigma x_{0}(t)\right) \mathrm{d} t \\
& \leq \frac{1}{p}\left\|\varsigma x_{0}\right\|^{p}-\lambda \int_{\Omega} F\left(t, \varsigma x_{0}(t)\right) \mathrm{d} t \\
& \leq \frac{\varsigma^{p}}{p}\left\|x_{0}\right\|^{p}-\frac{2 \lambda \varrho \varsigma^{p}}{\vartheta-p} \int_{\Omega}\left|x_{0}(t)\right|^{p} \mathrm{~d} t+\frac{2 \lambda \varrho|\Omega|}{\vartheta-p} K_{3}^{p}+\lambda \max _{s \in\left[0, K_{3}\right]} k_{1}(s) \int_{\Omega} k_{2}(t) \mathrm{d} t \\
& \leq\left(\frac{G^{p}}{p}-\frac{2 \lambda \varrho}{\vartheta-p}\right) \varsigma^{p}+\frac{2 \lambda \varrho|\Omega|}{\vartheta-p} K_{3}^{p}+\lambda \max _{s \in\left[0, K_{3}\right]} k_{1}(s) \int_{\Omega} k_{2}(t) \mathrm{d} t .
\end{aligned}
$$

Notice that $\varrho>\frac{(\vartheta-p) G^{p}}{2 \lambda p}$, we can get that $I\left(\varsigma x_{0}\right) \rightarrow-\infty$ as $\varsigma \rightarrow \infty$. Hence (3) of Lemma 3.2 holds.

Finally, note that $I(0)=0$, while for critical point $x, I(x) \geq \sigma>0$. So $x$ is a nontrivial weak solution of BVP (1.2). The proof is completed.

We then use Lemma 3.3 to show the multiplicity of solutions to BVP (1.2).

Theorem 3.3 Assume that $\left(A_{0}\right)$ and $\left(A_{1}\right)$ hold. Suppose also that there exist $r>0$ and $\omega \in E_{0}^{\alpha, p}$ such that $\frac{1}{p}\|\omega\|^{p}>r$ and

$$
\lambda_{l}=\frac{\left.\left.\frac{1}{p} \int_{0}^{T}\right|_{0} D_{t}^{\alpha} \omega(t)\right|^{p} \mathrm{~d} t}{\int_{0}^{T} F(t, \omega(t)) \mathrm{d} t}<\frac{r}{\int_{0}^{T} \max _{t \leq G \sqrt[p]{p r}} F(t, x) \mathrm{d} t}=\lambda_{r} .
$$

Then, for $\lambda \in\left(\lambda_{l}, \lambda_{r}\right), B V P(1.2)$ admits at least three weak solutions, which are critical points of the functional $I: E_{0}^{\alpha, p} \rightarrow \mathbb{R}$.

Proof As far as we know, $E_{0}^{\alpha, p}$ is a real reflexive Banach space. We consider the functionals $\Phi: E_{0}^{\alpha, p} \rightarrow \mathbb{R}$ and $\Psi: E_{0}^{\alpha, p} \rightarrow \mathbb{R}$ defined, respectively, by

$$
\Phi(x)=\frac{1}{p} \int_{0}^{T}\left|{ }_{0} D_{t}^{\alpha} x(t)\right|^{p} \mathrm{~d} t
$$

and

$$
\Psi(x)=\int_{0}^{T} F(t, x(t)) \mathrm{d} t
$$


According to the condition of $\left(A_{0}\right)$, we can know that $\Phi, \Psi$ are well defined and Gâteaux differentiable functionals whose Gâteaux derivatives at the point $x \in E_{0}^{\alpha, p}$ are the functionals $\Phi^{\prime}(x), \Psi^{\prime}(x) \in\left(E_{0}^{\alpha, p}\right)^{*}$, given by

$$
\Phi^{\prime}(x) y=\int_{0}^{T} \phi_{p}\left({ }_{0} D_{t}^{\alpha} x(t)\right)_{0} D_{t}^{\alpha} y(t) \mathrm{d} t
$$

and

$$
\Psi^{\prime}(x) y=\int_{0}^{T} f(t, x(t)) y(t) \mathrm{d} t
$$

for every $y \in E_{0}^{\alpha, p}$. For $\left\{x_{n}\right\}, x \in E_{0}^{\alpha, p}, x_{n} \rightarrow x$ in $E_{0}^{\alpha, p}$ as $n \rightarrow \infty$, by Lemma 2.2, we get that $\left\{x_{n}\right\}$ converges uniformly to $x$ in $C([0, T], \mathbb{R})$. Hence

$$
\begin{aligned}
\limsup _{n \rightarrow \infty} \Psi\left(x_{n}\right) & \leq \int_{0}^{T} \limsup _{n \rightarrow \infty} F\left(t, x_{n}\right) \mathrm{d} t \\
& =\int_{0}^{T} F(t, x) \mathrm{d} t \\
& =\Psi(x)
\end{aligned}
$$

which implies that $\Psi$ is sequentially weakly upper semicontinuous.

By $\left(A_{0}\right)$, one can get $F\left(t, x_{n}\right) \rightarrow F(t, x)$ as $n \rightarrow \infty$. By the Lebesgue control convergence theorem, $\Psi^{\prime}\left(x_{n}\right) \rightarrow \Psi^{\prime}(x)$ strongly, which implies that $\Psi^{\prime}: E_{0}^{\alpha, p} \rightarrow E_{0}^{\alpha, p}$ is strongly continuous on $E_{0}^{\alpha, p}$, that is, $\Psi^{\prime}$ is a compact operator.

From $\left(A_{0}\right)$, it is standard to see that $\Phi: E_{0}^{\alpha, p} \rightarrow \mathbb{R}$ is sequentially weakly lower semicontinuous, coercive, and its derivative $\Phi^{\prime}$ admits a continuous inverse on $\left(E_{0}^{\alpha, p}\right)^{*}$.

Moreover, it is clear that $\inf _{x \in E_{0}^{\alpha, p}} \Phi(x)=\Phi(0)=\Psi(0)=0$.

Put $r>0$, for $x \in E_{0}^{\alpha, p}$ with $\Phi(x)=\frac{1}{p}\|x\|^{p} \leq r$, then $\|x\|_{\infty}^{p} \leq G^{p}\|x\|^{p} \leq p G^{p} r$, we can get

$$
\begin{aligned}
\left\{x \in E_{0}^{\alpha, p}: \Phi(x) \leq r\right\} & \subseteq\left\{x: \frac{1}{p G^{p}}\|x\|_{\infty}^{p} \leq r\right\} \\
& =\left\{x: \max _{t \in[0, T]}|x(t)| \leq G \sqrt[p]{p r}\right\} .
\end{aligned}
$$

Hence, using $\lambda<\lambda_{r}$, we have

$$
\begin{aligned}
\sup _{\Phi(x) \leq r} \Psi(x) & =\sup _{\Phi(x) \leq r} \int_{0}^{T} F(t, x(t)) \mathrm{d} t \\
& \leq \int_{0}^{T} \max _{|x| \leq G p \sqrt[p]{p r}} F(t, x) \mathrm{d} t \\
& <\frac{r}{\lambda} .
\end{aligned}
$$

On the other hand, by $\lambda>\lambda_{l}$, we get

$$
\Psi(\omega)=\int_{0}^{T} F(t, \omega(t)) \mathrm{d} t>\frac{\Phi(\omega)}{\lambda} .
$$


Thus

$$
\frac{\Psi(\omega)}{\Phi(\omega)}>\frac{1}{\lambda}>\frac{\sup \{\Psi(x): \Phi(x) \leq r\}}{r}
$$

and (1) of Lemma 3.3 holds.

Then, let us verify that, for $\lambda \in\left(\lambda_{l}, \lambda_{r}\right), \Phi-\lambda \Psi$ is coercive. For $x \in E_{0}^{\alpha, p}$, we obtain

$$
\begin{aligned}
\Phi(x)-\lambda \Psi(x) & =\frac{1}{p} \int_{0}^{T}\left|{ }_{0} D_{t}^{\alpha} x(t)\right|^{p} \mathrm{~d} t-\lambda \int_{0}^{T} F(t, x(t)) \mathrm{d} t \\
& \geq \frac{1}{p}\|x\|^{p}-\lambda\left(\int_{0}^{T} \eta_{1}(t)|x|^{\theta} \mathrm{d} t+\int_{0}^{T} L_{1} \mathrm{~d} t\right) \\
& \geq \frac{1}{p}\|x\|^{p}-\lambda G^{\theta}\|x\|^{\theta} \int_{0}^{T} \eta_{1}(t) \mathrm{d} t-\lambda L_{1} T .
\end{aligned}
$$

Notice that $0 \leq \theta<p$, so $\Phi(x)-\lambda \Psi(x) \rightarrow+\infty$ as $\|x\| \rightarrow+\infty$, that is, $\Phi-\lambda \Psi$ is coercive. Obviously, (2) in Lemma 3.3 holds.

Then, by Lemma 3.3, we claim that, for each $\lambda \in\left(\lambda_{l}, \lambda_{r}\right), I: E_{0}^{\alpha, p} \rightarrow \mathbb{R}$ given by $I=\Phi-$ $\lambda \Psi$ has at least three distinct critical points in $E_{0}^{\alpha, p}$, that is, BVP (1.2) has at least three solutions.

\section{Acknowledgements}

The authors express their heartfelt thanks to the editors and referees who have provided some important suggestions.

\section{Funding}

The article is supported by the National Natural Science Foundation of China (Grant No.: 11572148, 11872201).

\section{Availability of data and materials}

This paper focuses on theoretical analysis, not involving experiments and data.

\section{Competing interests}

The authors declare that they have no competing interests.

\section{Authors' contributions}

The authors have equal contributions to each part of this paper. All authors read and approved the final manuscript.

\section{Publisher's Note}

Springer Nature remains neutral with regard to jurisdictional claims in published maps and institutional affiliations.

Received: 14 February 2020 Accepted: 19 March 2020 Published online: 30 March 2020

\section{References}

1. Zhou, Y.: Basic Theory of Fractional Differential Equations. World Scientific, Singapore (2014)

2. Pang, D., Jiang, W., Liu, S., Du, J.: Stability analysis for a single degree of freedom fractional oscillator. Phys. A 523, 498-506 (2019)

3. Oldham, K.: Reactional differential equations in electrochemistry. Adv. Eng. Softw. 41(1), 9-12 (2010)

4. Liu, F., Burrage, K.: Novel techniques in parameter estimation for fractional dynamical models arising from biological systems. Comput. Math. Appl. 62(3), 822-833 (2011)

5. Meng, X., Wang, L., Zhang, T.: Global dynamics analysis of a nonlinear impulsive stochastic chemostat system in a polluted environment. J. Appl. Anal. Comput. 6, 865-875 (2016)

6. Zhang, X., Liu, L., Wu, Y.: The uniqueness of positive solution for a singular fractional differential system involving derivatives. Commun. Nonlinear Sci. Numer. Simul. 18, 1400-1409 (2013)

7. Torres, C.: Existence of solutions for perturbed fractional Hamiltonian systems. JFCA 62-70 (2015)

8. Agarwal, R.P., Ertem, T., Zafer, A.: Asymptotic integration of second-order nonlinear delay differential equations. Appl. Math. Lett. 48, 128-134 (2015)

9. Bai, Z., Zhang, Y.: Solvability of fractional three-point boundary value problems with nonlinear growth. Appl. Math. Comput. 218, 1719-1725 (2011)

10. Zhang, $X$. ., Liu, L., Wu, Y.: Multiple positive solutions of a singular fractional differential equation with negatively perturbed term. Math. Comput. Modelling 55, 1263-1274 (2012) 
11. Dogan, A.: On the existence of positive solutions for the second-order boundary value problem. Appl. Math. Lett. 49, 107-112 (2015)

12. Chen, T., Liu, W.: An anti-periodic boundary value problem for the fractional differential equation with a $p$-Laplacian operator. Appl. Math. Lett. 25, 1671-1675 (2012)

13. Wang, Y., Liu, L., Wu, Y.: Positive solutions for a nonlocal fractional differential equation. Nonlinear Anal. 74, 3599-3605 (2011)

14. Zhang, X., Liu, L., Wu, Y.: The eigenvalue problem for a singular higher order fractional differential equation involving fractional derivatives. Appl. Math. Comput. 218, 8526-8536 (2012)

15. Jiang, W.: The existence of solutions to boundary value problems of fractional differential equations at resonance. Nonlinear Anal. 74, 1987-1994 (2011)

16. Qiao, Y., Zhou, Z.: Existence of solutions for a class of fractional differential equations with integral and anti-periodic boundary conditions. Bound. Value Probl. 2017, 11 (2017). https://doi.org/10.1186/s13661-016-0745-x

17. Francesconi, M., Mugnai, D.: The fractional Hartree equation without the Ambrosetti-Rabinowitz condition. Nonlinear Anal. Real World Appl. 33, 363-375 (2017)

18. Heidarkhani, S., Salari, A., Caristi, G., Barilla, D.: Perturbed nonlocal fourth order equations of Kirchhoff type with Navier boundary conditions. Bound. Value Probl. 2017, 86 (2017). https://doi.org/10.1186/s13661-017-0817-6

19. Jiao, F., Zhou, Y.: Existence results for fractional boundary value problem via critical point theory. Internat. J. Bifur. Chaos 22, 1250086 (2012)

20. Zhao, Y., Tang, L.: Multiplicity results for impulsive fractional differential equations with $p$-Laplacian via variational methods. Bound. Value Probl. 2017, 123 (2017). https://doi.org/10.1186/s13661-017-0855-0

21. Chen, T., Liu, W: Solvability of fractional boundary value problem with $p$-Laplacian via critical point theory. Bound. Value Probl. 2016, 75 (2016). https://doi.org/10.1186/s13661-016-0583-x

22. Podlubny, l.: Fractional Differential Equations. Mathematics in Science and Engineering, vol. 198. Academic Press. New Tork (1999)

23. Kilbas, A., Srivastava, H., Trujillo, J.: Theory and Applications of Fractional Differential Equations. Elsevier, Amsterdam (2006)

24. Mawhin, J., Willem, M.: Critical Point Theory and Hamiltonian Systems. Springer, New York (1989)

25. Rabinowitz, P.H.: Minimax Methods in Critical Point Theory with Applications to Differential Equations. Am. Math. Soc., Providence (1986)

26. Bonanno, G., Marano, S.A.: On the structure of the critical set of nondifferentiable functionals with a weak compactness condition. Appl. Anal. 89, 1-10 (2010)

27. Jia, M., Liu, X:: Multiplicity of solutions for integral boundary value problems of fractional differential equations with upper and lower solutions. Appl. Math. Comput. 232, 313-323 (2014)

28. Lian, H.: Boundary value problems for nonlinear ordinary differential equations on infinite intervals. Doctoral thesis (2007)

\section{Submit your manuscript to a SpringerOpen ${ }^{\circ}$ journal and benefit from:}

- Convenient online submission

- Rigorous peer review

- Open access: articles freely available online

- High visibility within the field

- Retaining the copyright to your article

Submit your next manuscript at $\gg$ springeropen.com 